\title{
SÍNDROME VESTIBULAR POR ACCIDENTE CEREBROVASCULAR ASOCIADO A HIPOTIROIDISMO SECUNDARIO
}

\author{
Erwin Esparza-Rangel, ${ }^{\star}$ Est. $_{1-2}$, Diego Noé Rodríguez-Sánchez, Esp., M.Sc. (c) ${ }_{3}$, Edwin Henry \\ Benavidez-Insignares, Esp. ${ }_{2}$
}
${ }_{1}$ Facultad de Medicina Veterinaria y Zootecnia, Universidad Cooperativa de Colombia, sede Bucaramanga, Colombia Clínica Veterinaria Dover, Bogotá, Colombia
${ }_{3}$ Facultad de Medicina Veterinaria y Zootecnia, UNESP, Botucatu, Brasil

Recibido: 5 de octubre del 2013 Aprobado: 3 de diciembre del 2013

*Autor de correspondencia: Erwin Esparza Rangel, Facultad de Medicina Veterinaria y Zootecnica, Universidad Cooperativa de Colombia, Bucaramanga, Colombia, calle 31 n. 33A-51, oficina 602. Teléfono: 6458500 ext. 7072. Correo electrónico: erwin.esparza@campusucc.edu.co

Cómo citar este artículo: Esparza-Rangel E., Rodríguez-Sánchez DN, Benavidez-Insignares EH. Síndrome vestibular por accidente cerebrovascular asociado a hipotiroidismo secundario. Spei Domus. 2013; 9(19): 57-63.

Resumen. El síndrome vestibular es una patología asociada a múltiples causas que afecta a perros de cualquier edad, sexo y raza. El tratamiento y el pronóstico dependerán siempre de la causa primaria y de la localización de la lesión. En este reporte se expone el caso de un paciente canino criollo que fue llevado a la Clínica Veterinaria Dover por presentar inclinación de la cabeza hacia el lado izquierdo, nistagmo horizontal, ataxia y caídas ocasionales hacia el mismo lado. Se realizaron exámenes paraclínicos (cuadro hemático, fosfatasa alcalina, glicemia, colesterol, triglicéridos) así como medición de la presión arterial y ecografía abdominal. Los resultados se relacionaron con hipotiroidismo e hiperadrenocorticismo, lo cual se confirmó posteriormente mediante un perfil tiroideo y medición de cortisol en sangre, respectivamente. Con base en esto se realizó resonancia magnética donde se encontró una estructura compatible con accidente cerebrovascular isquémico en el hemisferio cerebelar izquierdo, al cual se le atribuyó la aparición de los síntomas vestibulares. Finalmente, se concluyó que el accidente cerebrovascular se presentó como consecuencia de hipotiroidismo secundario.

Palabras clave: enfermedad vascular, enfermedad vestibular, hiperadrenocorticismo, hipotiroidismo.

\section{Vestibular Syndrome Caused by a Cerebrovascular} Accident Associated with Secondary Hypothyroidism

\begin{abstract}
Vestibular syndrome is a pathology associated with multiple causes and affects dogs of any age, gender or breed. The treatment and prognosis will always depend on the primary cause and the location of the lesion. This report addresses the case of a Creole canine patient brought to the Clínica Veterinaria Dover because it inclined its head towards the left side, had horizontal nystagmus and ataxia along with occasional falls towards that same side. Laboratory exams were carried out (blood work, alkaline phosphatase, glycemia, cholesterol, triglycerides) along with the use of medication for blood pressure and an abdominal ultrasound. The results are associated with hypothyroidism and hyperadrenocorticism, which was subsequently confirmed by using a thyroid profile and measuring blood cortisol, respectively. Based on this, magnetic resonance was performed, in which a structure was found that was compatible with an ischemic cerebrovascular accident in the left cerebellar hemisphere, to which the appearance of the vestibular symptoms was attributed. The conclusion was that the cerebrovascular accident occurred as a result of secondary hypothyroidism.
\end{abstract}

Keywords: vascular disease, vestibular disease, hyperadrenocorticism, hypothyroidism.

\section{Síndrome vestibular por acidente cerebrovascular associado a hipotireoidismo secundário}

Resumo. A síndrome vestibular é uma patologia associada a múltiplas causas que afeta a cachorros de qualquer idade, sexo e raça. $\mathrm{O}$ tratamento e o prognóstico dependerão sempre da causa primária e da localização da lesão. Neste relatório, expõe-se o caso de um paciente canino crioulo que foi levado à Clínica Veterinária Dover por apresentar inclinação da cabeça para o lado esquerdo, nistagmo horizontal, ataxia e quedas ocasionais para o mesmo lado. Realizaram-se exames de laboratório (quadro hemático, fosfatase alcalina, glicemia, colesterol, triglicerídeos), bem como medição da pressão arterial e ecografia abdominal. Os resultados se relacionaram com hipotireoidismo e hiperadrenocorticismo, o qual se confirmou posteriormente mediante um perfil tireóideo e medição de cortisol em sangue, respectivamente. Com base nisso, realizou-se ressonância magnética na qual se encontrou uma estrutura compatível com acidente cerebrovascular isquêmico no hemisfério cerebelar esquerdo, ao qual foi atribuído o aparecimento dos sintomas vestibulares. Finalmente, concluiu-se que o acidente cerebrovascular se apresentou como consequência de hipotireoidismo secundário.

Palavras-chave: doença vascular, doença vestibular, hiperadrenocorticismo, hipotireoidismo. 


\section{Introducción}

De una manera simplificada se puede decir que el sistema vestibular se compone de porciones centrales y periféricas [1]. El sistema vestibular periférico está compuesto por receptores sensoriales que se localizan en el laberinto membranoso del oído interno dentro de la porción petrosa del hueso temporal del cráneo y la porción vestibular del nervio vestibulococlear (viII par craneal), el cual transporta información desde dichos receptores hasta el tronco del encéfalo $[1,2]$. Las estructuras vestibulares centrales comprenden los núcleos vestibulares del tronco encefálico y las vías de la médula oblonga y el lóbulo floculonodular del cerebelo $[1,3]$.

Los núcleos vestibulares del tronco encefálico reciben señales y envían mensajes a varias partes del cuerpo para cumplir las siguientes funciones [3]:

- A la médula espinal, para ejercer un efecto facilitador sobre los músculos extensores de las extremidades ipsilaterales.

- A los ojos, para controlar los músculos involucrados en los movimientos del globo ocular.

- Al prosencéfalo, para la percepción consciente de la posición (propiocepción).

- Al cerebelo, para mantener la coordinación de los ojos, cuello, cuerpo y extremidades en relación con los movimientos de la cabeza.

- A la formación reticular en el tronco encefálico, área responsable del vómito y el mareo.

En pocas palabras, el sistema vestibular es, en gran parte, responsable del mantenimiento de la orientación de los animales $[4,5]$. Por tanto, la sintomatología que puede ser observada en el síndrome vestibular incluye inclinación de la cabeza, movimientos en círculo, ataxia, caídas, movimientos de rodeo y nistagmos $[1,4-6]$.

La inclinación de la cabeza se describe como una rotación de la cabeza respecto al atlas $(\mathrm{C} 1)$, de manera que uno de los pabellones auriculares está por debajo del otro. El ladeo de la cabeza es indicativo de enfermedad vestibular y es el signo más constante de un déficit vestibular unilateral [6]. Tanto en la enfermedad central como periférica, la inclinación de la cabeza, el ladeo y cualquier déficit en cualquier extremidad típicamente ocurren ipsilaterales a la lesión $[1,4,6]$. Un animal que sufre enfermedad bilateral del sistema vestibular no presenta ladeo de la cabeza ni nistagmo espontáneo o posicional [6].

El nistagmo se define como una oscilación rítmica e involuntaria de los globos oculares $[1,6]$. Cuando el sis- tema vestibular está alterado, los ojos tienen una tendencia espontánea a moverse en dirección de la lesión (fase lenta o patológica) y, a través de un mecanismo cerebral, los ojos son rápidamente devueltos a su ubicación inicial (fase rápida, correctiva o compensatoria) $[4,6]$. El nistagmo de sacudida o en resorte, en el que los movimientos oculares tienen una fase lenta en una dirección y una recuperación rápida en la dirección contraria, se observa frecuentemente en enfermedad vestibular $[1,6]$.

El nistagmo que se observa cuando la cabeza está en posición estática normal se llama nistagmo espontáneo o nistagmo de reposo, mientras el que está inducido por movimientos de la cabeza hacia una posición inusual se llama nistagmo posicional $[4,6]$. El nistagmo en un paciente con enfermedad vestibular periférica es siempre horizontal o bien rotatorio, $y$, aunque la intensidad del nistagmo puede variar si se sujeta la cabeza en diferentes posiciones, la dirección no se va a modificar. En animales con enfermedades vestibulares centrales el nistagmo puede ser horizontal, rotatorio o vertical, y su dirección puede cambiar a medida que se modifica la posición de la cabeza [1].

El diagnóstico diferencial de un animal con síndrome vestibular varía considerablemente dependiendo de si se determina que los déficits vestibulares son centrales o si tiene un origen periférico [6]. Se han planteado diferentes mecanismos por los cuales se puede producir enfermedad vestibular periférica o central $[1,6,7]$ :

- Causas de enfermedad vestibular periférica:

- Enfermedades inflamatorias/infecciosas: otitis media/interna (es la causa más común en perros y gatos), pólipos inflamatorios nasofaríngeos (pueden producir otitis media secundaria).

- Traumatismos (de la porción petrosa del hueso temporal): producen compresión directa del nervio vestibulococlear (NC. VIII) y lesión del laberinto óseo y membranoso.

- Congénito: síndrome vestibular congénito (común en perros de raza pastor alemán, doberman pinscher, akita, cocker spaniel, fox terrier pelo liso, beagle y terrier del tibet y en gatos de raza siamés, burmés, tonkinés y persa)

- Enfermedades metabólicas: hipotiroidismo

- Agentes ototóxicos: antibióticos aminoglucósidos (estreptomicina, gentamicina, neomicina, etc.), antineoplásicos (vincristina, cisplatino, vinblastina, etc.), yodóforos tópicos y clorhexidina

- Idiopático: enfermedad vestibular geriátrica canina y síndrome vestibular idiopático felino 
- Neoplasias: tumores del oído medio o interno (adenomas o adenocarcinomas de glándula ceruminosa) o tumores del nervio vestibulococlear (infrecuentes).

- Causas de enfermedad vestibular central:

- Enfermedades vasculares: accidentes cerebrovasculares (ACV)

- Enfermedades inflamatorias/infecciosas: encefalomielitis por moquillo, meningoencefalomielitis granulomatosa y encefalitis necrotizante del yorkshire en perros y peritonitis infecciosa felina en gatos son las causas más comunes.

- Traumatismos del tronco encefálico.

- Anomalías congénitas: quistes aracnoideos (producen compresión del cerebelo y tronco encefálico) y síndrome de malformación occipital caudal.

- Enfermedades metabólicas: hipotiroidismo (arteroscleromas).

- Tóxicos: intoxicación por metronidazol

- Nutricionales: deficiencia de tiamina

- Neoplasias: se destacan neoplasias extraparenquimatosas del cuarto ventrículo y la base del tronco encefálico. En perros meningiomas y tumor de plexos coroideos y en gatos meningiomas y linfomas en la fosa caudal, principalmente.

- Degenerativas: enfermedades de almacenamiento lisosomal.

Son múltiples las causas que pueden producir enfermedad vestibular, razón por la cual todo paciente que ingrese con signos asociados a dicha enfermedad se convierte en un reto para el médico veterinario, pues es su responsabilidad identificar la causa para establecer un pronóstico y así mismo el plan para seguir.

\section{Caso clínico}

El día 18 de abril del 2013 ingresó a la Clínica Veterinaria Dover (Bogotá, Colombia) un canino criollo, macho (entero), de aproximadamente 14 años de edad, con un peso de 10,5 kg de peso (P). Presentó inclinación marcada de la cabeza hacia el lado izquierdo, nistagmo horizontal espontaneo, ataxia y caídas ocasionales por el lado izquierdo; todos de aparición aguda con un tiempo de evolución de 14 horas según reportó el propietario.

$\mathrm{Al}$ examen clínico presentó temperatura $(\mathrm{T})$ de $37,8^{\circ} \mathrm{C}$, frecuencia cardiaca $(\mathrm{Fc})$ de $148 \mathrm{lpm}$, frecuen- cia respiratorio $(\mathrm{Fr})$ : jadeo, membranas mucosas (MM) congestionadas, tiempo de llenado capilar (TLC) un segundo, condición corporal (cc) 3/5, estado de conciencia alerta, propiocepción presente. El propietario reportó que el paciente actualmente no recibía ningún tipo de medicación y que sólo había observado un leve aumento del consumo de agua durante los últimos 15 días. Al examen por sistemas se descartó otitis y se evidenció la presencia de comedones en la región abdominal junto con debilidad del tren posterior.

Como primera medida, el paciente fue hospitalizado y se le administró:

Líquidos de mantenimiento: $\mathrm{NaCl} 60 \mathrm{ml} / \mathrm{kg} /$ día, IV.

Diuréticos: furosemida $4 \mathrm{mg} / \mathrm{kg}$, IV (dosis inicial) y $2 \mathrm{mg} / \mathrm{kg}$ BID, IV (dosis de mantenimiento); seguido de manitol $500 \mathrm{mg} / \mathrm{kg}$, BID, IV.

Adicionalmente se administró oxigeno de soporte. El mismo día de ingreso se realizó perfil hematológico (tabla 1) y químicas sanguíneas (tabla 2). Debido a los hallazgos en el recuento plaquetario $\left(747.000 / \mathrm{mm}^{3}\right) \mathrm{y}$ la fosfatasa alcalina (1.819 U. I.) se sospechó de hiperadrenocorticismo, razón por la cual se realizó medición de presión arterial (tabla 3) y ecografía abdominal con énfasis en glándulas adrenales En ambas glándulas adrenales se observa un contorno conservado, un parénquima fino granular y aumento de tamaño predominante del polo caudal; de esto se concluye que existe adrenomegalia bilateral, que se observa comúnmente en pacientes con hiperadrenocorticismo dependiente de pituitaria. No se encontró evidencia de alteraciones en los demás órganos abdominales (figura 1). Así mismo, se hizo medición de TsH y T4 Total (tabla 4) debido al leve aumento de los triglicéridos.

Tabla 1. Hematología canina

\begin{tabular}{|c|c|c|}
\hline Prueba & Valor normal & Resultado \\
\hline Hematocrito (\%) & $37-52$ & 58,8 \\
\hline Hemoglobina (\%) & $12-18$ & 18,2 \\
\hline G. blancos $\left(\# / \mathrm{mm}^{3}\right)$ & $6.000-14.000$ & 11.100 \\
\hline Neutrófilos (\%) & $56-78$ & 87 \\
\hline Neutrófilos $\left(\# / \mathrm{mm}^{3}\right)$ & $4.000-10.600$ & 9.657 \\
\hline Linfocitos (\%) & $13-30$ & 13 \\
\hline Linfocitos $\left(\# / \mathrm{mm}^{3}\right)$ & $900-4.100$ & 1.443 \\
\hline Eosinófilos (\%) & $2-6$ & -- \\
\hline Eosinófilos $\left(\# / \mathrm{mm}^{3}\right)$ & $140-800$ & --- \\
\hline Plaquetas $\left(\# / \mathrm{mm}^{3}\right)$ & $200.000-400.000$ & 747.000 \\
\hline
\end{tabular}

Fuente: elaboración propia 
Tabla 2. Química sanguínea

\begin{tabular}{|l|l|l|}
\hline \multicolumn{1}{|c|}{ Prueba } & \multicolumn{1}{c|}{ Valor normal } & \multicolumn{1}{c|}{ Resultado } \\
\hline Creatinina (mg/dl) & $<1,5$ & 0,62 \\
\hline GPT - ALT (U.I.) & $5-80$ & 87,5 \\
\hline Fosfatasa Alcalina (U.I.) & $20-140$ & 1.819 \\
\hline Glicemia (mg/dl) & $60-140$ & 69 \\
\hline Colesterol (mg/dl) & $105-440$ & 236 \\
\hline Triglicéridos (mg/dl) & $<150$ & 152 \\
\hline
\end{tabular}

Fuente: elaboración propia

Tabla 3. Presión arterial

\begin{tabular}{|l|c|c|}
\hline \multicolumn{1}{|c|}{ Prueba } & Valor normal & Resultado \\
\hline Presión arterial sistólica & $115-135$ & 144 \\
\hline Presión arterial diastólica & $70-90$ & 95 \\
\hline Presión arterial media & $85-105$ & 121 \\
\hline
\end{tabular}

Fuente: elaboración propia

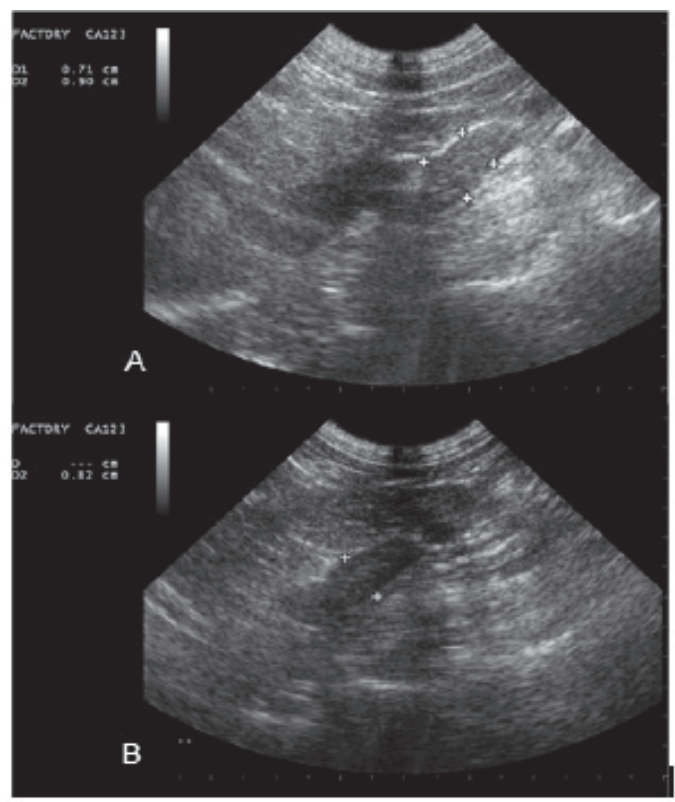

Figura 1. Ecografía abdominal. (A) Glándula Adrenal Derecha: polo craneal 0,71 cm, polo caudal 0,9 cm. (B) Glándula Adrenal Izquierda: polo craneal $0,76 \mathrm{~cm}$, polo caudal $0,82 \mathrm{~cm}$. Tamaño normal: hasta $0,7 \mathrm{~cm}$ de diámetro, siendo el polo caudal mayor que el craneal.

Fuente: elaboración propia

Con base en los resultados obtenidos - aumento de la fosfatasa alcalina, trombocitosis, hipertensión y adrenomegalia bilateral-, era un paciente con un perfil altamente sospechoso de padecer hiperadrenocorticismo; por esta razón se decidió realizar prueba de supresión con dexametasona a dosis baja (SDDB) (tabla 5).
De igual forma, se inició tratamiento sintomático antihipertensivo:

Inhibidores de la IECA: enalapril 0,5 mg/kg, BID, PO.

Bloqueadores de canales de calcio (BCC): flunarizina $0,5 \mathrm{mg} / \mathrm{kg}$. BID, PO.

Adicional a ello se administró melatonina $3 \mathrm{mg} /$ perro, SID, PO como antioxidante.

Tabla 4. Perfil tiroideo

\begin{tabular}{|l|c|c|}
\hline \multicolumn{1}{|c|}{ Prueba } & Valor normal & Resultado \\
\hline tsh canino $(\mathrm{ng} / \mathrm{ml})$ & $0,0-0,5$ & 0,05 \\
\hline T4 Total canino $(\mathrm{ug} / \mathrm{dl})$ & $1,32-2,9$ & 0,62 \\
\hline
\end{tabular}

Fuente: elaboración propia

Tres días después se obtuvieron los resultados del perfil tiroideo revelando una marcada disminución en la concentración de T4 Total y valores normales para la TSH - hallazgo compatible con hipotiroidismo secundario por supresión de las células tirotropas hipofisarias, siendo las causas más comunes hiperadrenocorticismo espontaneo (adquirido de forma natural) y síndrome eutiroideo enfermo- [8]. A partir de estos hallazgos se decidió suplementar la hormona tiroidea (levotiroxina sódica $0,022 \mathrm{mg} / \mathrm{kg}$, BID, PO).

Este mismo día se relaciona el síndrome vestibular a un ACV secundario al hipotiroidismo, razón por la cual se realizó resonancia magnética (RM) de cerebro (figura 2). Después de seis días de hospitalización, los signos asociados al síndrome vestibular habían disminuido - leve inclinación de la cabeza hacia el lado izquierdo, nistagmo horizontal espontaneo leve, ataxia leve, sin caídas-. Debido a la mejoría clínica, cuatro (4) días despues de su ingreso, el paciente fue dado de alta con la siguiente medicación para administración domiciliaria:

Enalapril 0,5 mg/kg, PO, BID, tiempo indefinido.

Flunarizina $0,5 \mathrm{mg} / \mathrm{kg}$, PO, BID, tiempo indefinido.

Levotiroxina sódica $0,022 \mathrm{mg} / \mathrm{kg}$, PO, BID, tiempo indefinido.

Melatonina $3 \mathrm{mg} /$ perro, PO, sID, tiempo indefinido.

Se realizó cita de control siete días después de la salida del paciente, y en el examen clínico se encontró $\mathrm{P}$ : 10,2 kg, T: 38, $4^{\circ} \mathrm{C}, \mathrm{Fc}: 128 \mathrm{lpm}, \mathrm{Fr}: 16 \mathrm{rpm}, \mathrm{mm}$ : rosadas, TLC: un segundo, CC. $3 / 5$ y estado de conciencia alerta.

En cuanto a los signos asociados al síndrome vestibular, persistía la inclinación leve de la cabeza hacía el lado izquierdo, el nistagmo horizontal fue casi imperceptible y 
no presentó ataxia; sin embargo, en estación presentó posición de base ancha y al desplazarse pasos hipermétricos.

Este mismo día se obtuvo el resultado de la prueba de SDDB (tabla 5) y con base en ello - supresión de cortisol a las cuatro horas y escape de la supresión a las ocho horas- se concluyó que el paciente padecía hiperadrenocorticismo dependiente de pituitaria; por esta razón se dio inicio al tratamiento domiciliario con trilostano $2 \mathrm{mg} / \mathrm{kg}$, PO, sID, por tiempo indefinido. A su vez se suspende la flunarizina y la demás medicación se continúa administrando a igual dosis y frecuencia.

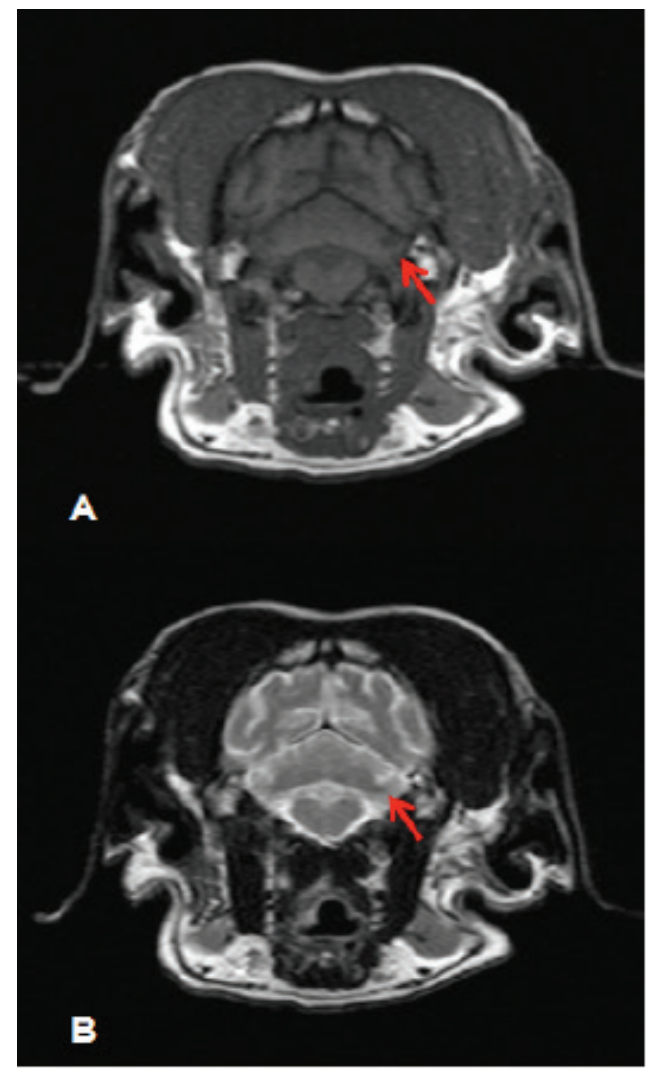

Figura 2. RM. (A) Vista Axial de Cerebro Ponderada en t1 a Nivel de los Hemisferios Cerebelares y Metencéfalo

Fuente: elaboración propia

Puede observarse en la figura 2 un área hipointensa (flecha roja) que señala un área hipointensa de forma redondeada a nivel de la base del hemisferio cerebelar izquierdo, que no genera efecto de masa. (B) Vista Axial de Cerebro Ponderada en t2 a Nivel de los Hemisferios Cerebelares y Metencéfalo: la flecha señala un área hiperintensa de forma redondeada, territorial, de tamaño pequeño, que no genera efecto de masa ni edema perilesional. Estas imágenes son compatibles con ACV isquémico regional. La lesión identificada en las imágenes anteriores coincide con la presentación clínica del paciente.

Se realizaron citas de control para realizar monitoreo de hiperadrenocorticismo e hipotiroidismo con el fin de ajustar la dosificación y la frecuencia de administración de los medicamentos.

Tabla 5. Prueba de supresión con dexametasona a dosis baja (SDDB)

\begin{tabular}{|l|l|l|}
\hline \multicolumn{1}{|c|}{ Prueba } & \multicolumn{1}{|c|}{ Valor normal } & Resultado \\
\hline Cortisol Basal (nmol/L) & $25-125$ & 94,1 \\
\hline Cortisol Post 4 horas (nmol/L) & 30 & 28,7 \\
\hline Cortisol Post 8 horas (nmol/L) & 30 & 32 \\
\hline
\end{tabular}

Fuente: elaboración propia

Finalmente, se estableció como diagnóstico definitivo Síndrome Vestibular Central por accidente cerebrovascular, el cual, con base en los hallazgos de laboratorio y la respuesta satisfactoria al tratamiento, se relacionó a hipotiroidismo secundario por supresión de las células tirotropas a causa de hiperadrenocorticismo hipofisario.

\section{Discusión}

El síndrome vestibular es una patología que se manifiesta mediante signos característicos que permiten al médico veterinario reconocer fácilmente su padecimiento a través del examen clínico; sin embargo, es secundario a múltiples causas, y, por tanto, su diagnóstico, así como el establecimiento de un tratamiento adecuado y del pronóstico, se hacen una tarea dispendiosa ya que estos van ligados siempre a la causa primaria que desencadena el problema.

Una de las posibles causas del síndrome vestibular es el hipotiroidismo, un trastorno endocrino relativamente frecuente en los perros [9], por lo cual constituye un importante diagnóstico diferencial en casos de enfermedad vestibular en perros adultos.

Desde el punto de vista clínico, se debe recordar que las hormonas tiroideas activan directa o indirectamente la termogénesis oxidativa y la actividad metabólica de la mayoría de los tejidos; por esto, precisamente, es por lo que su deficiencia puede dar lugar a múltiples manifestaciones clínicas - síntomas generales, cutáneos, cardiovasculares, reproductivos, nerviosos, etcétera $[9,10]-$, que no se presentan todas en el mismo animal ni con la misma intensidad, lo que explica la 
gran variedad de cuadros clínicos que pueden aparecer en esta enfermedad [9].

Los síntomas neurológicos en enfermedades endocrinas como el hipotiroidismo son, en muchos casos, el problema predominante [8]. Esto fue demostrado mediante un estudio retrospectivo realizado entre 1987 y 1992 a 66 perros hipotiroideos, en el que el 28,8\% (19 perros) presentaron únicamente manifestaciones neurológicas [11]. Los déficits neurológicos pueden implicar la participación del sistema nervioso periférico y el sistema nervioso central [12] debido a que el hipotiroidismo induce desmielinización segmentaria y axonopatía. Estos signos también pueden aparecer por el acumulo de mucopolisacáridos en el perineuro y el endoneuro, por enfermedad cerebrovascular (arterosclerosis), o el desarrollo de hiperlipidemia $[8,13]$.

El término enfermedad cerebrovascular hace referencia a cualquier anomalía del cerebro causada por un proceso patológico que comprometa la irrigación sanguínea [14-16]. Se ha reportado que es poco común la presentación de accidentes cerebrovasculares (ACV) en pequeños animales [15]; sin embargo, constituyen la presentación clínica más común de enfermedad cerebrovascular en perros $[16,17]$. Los perros de edad avanzada, con fallo renal, hiperadrenocorticismo, hipotiroidismo o hipertensión de cualquier etiología tienen una especial predisposición a padecer estos trastornos [13, 18]. Por su parte, un estudio realizado a 42 perros entre los años 2007 y 2009 demuestra que el diagnóstico de ACV en perros por hiperadrenocorticismo y por hipotiroidismo fue de $14 \%$ y $2,5 \%$, respectivamente [17].

Los ACV se dividen en dos grupos: infarto isquémico e infarto hemorrágico [14, 15, 17]. El infarto isquémico puede presentarse como consecuencia de un trombo, una embolia o secundario a anormalidades de los vasos sanguíneos o cualquier otra causa que comprometa la irrigación arterial o venosa del cerebro [14, 15].

Los ACV y, en general, las enfermedades del sistema nervioso central son cada vez más reconocidas debido al avance de las técnicas de imagenología como es el caso de la RM $[14,15]$ y la tomografía computarizada (TC) [14]. Estos exámenes no invasivos ofrecen mucha información respecto a la localización y la extensión de las lesiones cerebrales [14].

Según este orden de ideas, tanto la RM como la TC también son útiles para confirmar la existencia de tumores de la glándula hipofisaria, los cuales se encuentran presentes entre el 80 y el $85 \%$ de los perros con hiperadrenocorticismo espontáneo $[8,19]$. El resto de los casos de hiperadrenocorticismo espontáneo (15-20\%) se relacionan con tumores adrenocorticales $[8,19]$.

Los tumores hipofisarios pueden clasificarse como microtumores ( $<10 \mathrm{~mm}$ de diámetro) y macrotumores $(>10 \mathrm{~mm}$ de diámetro) $[8,19]$. Aproximadamente entre el 80 y el 90\% de los perros presentan microtumores y sólo entre el 10 y el $20 \%$, macrotumores $[8,19]$. Del total de perros con tumores hipofisarios, el $50 \%$ presentan tumores inferiores a $3 \mathrm{~mm}$ de diámetro [8].

Se ha reportado que la TC permite únicamente la visualización de macrotumores y que mediante RM, por brindar un contraste superior, es preciso identificar tumores más pequeños [19]; sin embargo, en el presente caso no fue posible evidenciar tumores hipofisarios, lo cual lleva a pensar que el paciente tiene un microadenoma de tamaño inferior a $3 \mathrm{~mm}$ de diámetro, lo cual impidió que fuera visible al momento de la RM.

Finalmente, respecto a los ACV, cabe resaltar que no existe resolución de estos y que el tratamiento de los animales afectados deberá ir encaminado a la prevención de las lesiones secundarias y de las complicaciones [20]. El pronóstico dependerá del tipo y la localización de la lesión, así como de la gravedad del daño neurológico, de la aparición de complicaciones secundarias y la causa subyacente de la enfermedad vascular [16]; esto sustenta el hecho de que el paciente se estabilizó con la terapia diurética que se utilizó para disminuir la presión intracraneal y el tratamiento antihipertensor antes de tratar la causa primaria.

\section{Conclusiones}

El reconocimiento de la enfermedad vestibular en perros se basa en un adecuado examen neurológico. Sin embargo, es una enfermedad compleja que requiere del conocimiento anatómico y fisiológico del sistema vestibular para comprender la aparición de los signos clínicos y, de igual forma, los mecanismos por los cuales diferentes patologías pueden producirlo.

Enfermedades endocrinas como el hiperadrenocorticismo pueden alterar procesos fisiológicos de la glándula tiroides que inducen de forma secundaria al desarrollo de hipotiroidismo, que cursa con una sintomatología muy variada, pudiendo en algunos casos llegar a desarrollar únicamente manifestaciones neurológicas.

La RM es una técnica útil para diagnosticar lesiones en el sistema nervioso central como los ACv. No obstante, la única forma de llegar a identificar su verda- 
dero origen y así mismo llegar a un diagnóstico definitivo, en estos casos, es mediante el hallazgo de la lesión misma al realizar necropsia al paciente.

\section{Referencias}

[1] Nelson RW, Couto CG. Inclinación de la cabeza. En: Nelson RW, Couto CG, editores. Medicina interna de pequeños animales. 4a ed. Barcelona: Elsevier; 2010. p. 1047-53.

[2] LeCouteur RA, Vernau KM. Feline vestibular disorders. Part I: anatomy and clinical signs. J Feline Med Surg. 1999; 1(2): 71-80.

[3] Kent M, Platt SR, Schatzberg SJ. The neurology of balance: function and dysfunction of the vestibular system in dogs and cats. Vet J. 2010; 185(3): 247-58.

[4] Bagley RS. Enfermedad vestibular en perros y gatos. En: Bonagura JD, Twedt DC, editores. Kirk Terapéutica veterinaria actual. 14a ed. Barcelona: Elsevier; 2010. p. 1097-101.

[5] Garosi LS. Vestibular disease in dogs and cats. In practice. $2007 ; 29(3): 151-7$.

[6] Muñana KR. Inclinación de la cabeza y nistagmo. En: Platt SR, Olby NJ, editores. Manual de neurología en pequeños animales. 3a ed. Barcelona: Lexus; 2012. 211-33.

[7] Montoliu P. Síndrome vestibular. En: Morales C, Montoliu P, editores. Neurología canina y felina. 1a ed. Barcelona: Multimédica; 2012. 413-29.

[8] Nelson RW, Couto CG. Alteraciones de la glándula tiroidea. En: Nelson RW, Couto CG, editores. Medicina interna de pequeños animales. 4a ed. Barcelona: Elsevier; 2010. p. 724-63.

[9] Marca MC, Loste A, Sanz MC, Sáez T, Verde MT, et al. Hipotiroidismo canino: revisión y actualización de su diagnóstico. Clínica Veterinaria de Pequeños Animales (Avepa). 1999; 16(2): 111-7.
[10] Scott-Moncrieff JC. Signos clínicos y enfermedades concurrentes en el hipotiroidismo en perros y gatos. Vet Clin Small Anim. Jul 2007; 37(4): 709-22.

[11] Panciera DL. Hypothyroidism in dogs: 66 cases (19871992). J Am Vet Med Assoc. 1994; 204(5): 761-7.

[12] Vitale CL, Olby NJ. Neurologic dysfunction in hypothyroid, hyperlipidemic Labrador Retrievers. J Vet Intern Med. 2007; 21(6): 1316-22.

[13] Rossmeisl JH. Vestibular disease in dogs and cats. Vet Clin North Am Small Anim Pract. 2010; Ene 40(1): 81-100.

[14] Babicsak VR, Machado VMV, dos Santos DR, Zardo KM, Campos LA. Aspectos de infartos isquêmicos em cães na tomografia computadorizada e ressonância magnética. Vet. e Zootec. 2011; 18(2): 208-13.

[15] McConnell JF, Garosi LS, Platt SR. Magnetic resonance imaging findings of presumed cerebellar cerebrovascular accident in twelve dogs. Vet Radiol Ultrasound. Ene-Feb 2005; 46(1): 1-10.

[16] Garosi LS, Platt SR. Tratamiento de la enfermedad cerebrovascular. En: Bonagura JD, Twedt DC, editores. Kirk Terapéutica veterinaria actual. 14a ed. Barcelona: Elsevier; 2010. p. 1074-7.

[17] Carvalho CF, Andrade JP, Diniz SA. Small breed dogs with confirmed stroke: concurrent diseases and sonographic findings. Arq Bras Med Vet Zootec. 2012; 64(5): 1177-83.

[18] Nelson RW, Couto CG. Trastornos intracraneales. En: Nelson RW, Couto CG, editores. Medicina interna de pequeños animales. 4a ed. Barcelona: Elsevier; 2010. p. 1019-26.

[19] Peterson ME. Diagnosis of hyperadrenocorticism in dogs. Clin Tech Small Anim Pract. Feb 2007; 22(1): 2-11.

[20] Wessmann A, Chandler K, Garosi L. Ischaemic and haemorrhagic stroke in the dog. Vet J. Jun 2009; 180(3): 290-303. 